\title{
"I Don't Want to Spend the Rest of my Life Only Going to a Gender Wellness Clinic": Healthcare Experiences of Patients of a Comprehensive Transgender Clinic
}

\author{
Joy L. Lee, PhD, MS ${ }^{1,2}$ (D, Monica Huffman, BA ${ }^{2}$, Nicholas A. Rattray, PhD ${ }^{1,2,3}$, \\ Jennifer L. Carnahan, MD, MPH ${ }^{1,2}$, J. Dennis Fortenberry, MD, MS ${ }^{l}$, \\ Janine M. Fogel, $\mathrm{MD}^{1,4}$, Michael Weiner, $M D, M P H^{1,2,3}$, and Marianne S. Matthias, \\ $P h D^{1,2,3}$
}

\begin{abstract}
'Indiana University School of Medicine, Indianapolis, IN, USA; ${ }^{2}$ Regenstrief Institute, Inc., Indianapolis, IN, USA; ${ }^{3}$ Center for Health Information and Communication, U.S. Department of Veterans Affairs, Veterans Health Administration, Health Services Research and Development Service CIN 13416, Richard L. Roudebush VA Medical Center, Indianapolis, IN, USA; ${ }^{4}$ Gender Health Program, Eskenazi Health, Indianapolis, IN, USA.
\end{abstract}

BACKGROUND: Transgender individuals are less likely to have had a primary care visit in the last year than cisgender individuals. While the importance of multidisciplinary clinics for transgender care has been established, little is known about the healthcare experiences of transgender patients with these clinics.

OBJECTIVE: To describe how patients experience transgender clinics and how these experiences compare to those experiences in other settings.

PARTICIPANTS: Twenty-one adult patients of a gender health program.

DESIGN AND APPROACH: Semi-structured interviews of transgender patients. The interviews focused on two domains: healthcare experiences and relationships with healthcare providers.

KEY RESULTS: Overall, transgender patients expressed a need for healthcare services, particularly for primary care, that are partially met by the comprehensive care clinic model. Limitations in access included the lack of willing providers, where the patients live, and long wait times for appointments. Participants recounted a range of experiences, both positive and negative, with providers outside of the transgender clinic, but only positive experiences to share about providers from the transgender clinic.

CONCLUSION: Outside specialty transgender settings, many patients had negative experiences with providers who were unwilling or unable to provide care. This study speaks to the need for primary care providers who can and will treat transgender patients, as well as the need for healthcare spaces that feel safe to transgender patents.

KEY WORDS: primary care; transgender health; qualitative research.

Received August 13, 2021

Accepted January 7, 2022

Published online February 2, 2022
J Gen Intern Med 37(13):3396-403 DOI: $10.1007 / \mathrm{s} 11606-022-07408-5$

(c) The Author(s) under exclusive licence to Society of General Internal Medicine 2022

\section{BACKGROUND}

Transgender adults report a higher burden of disability ${ }^{1}$, and have more chronic conditions, including human immunodeficiency virus, diabetes mellitus, and obesity, than cisgender adults. ${ }^{1-3}$ The disparities in mental health outcomes are also stark. Transgender individuals are more than twice as likely than cisgender individuals to be diagnosed with depression and to experience mental health distress. ${ }^{1,2}$ Despite evidence of greater medical needs, transgender patients often face unique barriers to accessing healthcare. The disparities extend beyond health itself, to primary care access and utilization. Transgender individuals are less likely to have had a primary care visit in the last year than cisgender individuals. ${ }^{1}$ Many cite fear of discrimination as a reason for delaying care. ${ }^{4}$

Specialty care clinics that cater specifically to the needs of gender-diverse adults are one suggested solution to the healthcare needs of this population. Multidisciplinary, comprehensive transgender health clinics may be able to provide "one-stop shop" care for patients in an affirming environment, reducing some of the barriers to care. ${ }^{5}$ While the importance of multidisciplinary healthcare providers in treating transgender patients has been established, 5,6 little is known about the healthcare experiences of transgender patients with these clinics, particularly surrounding primary care. A few studies have found high patient satisfaction with transgender-specific healthcare. ${ }^{7-9}$ However, there is a dearth of research about specific clinic features that satisfy patients. Even less is known about the healthcare needs of transgender people when seeking specialty care in a transgender clinic. Understanding the in-depth experiences and needs of patients is needed for 
designing effective interventions that address barriers to care. In this study, we sought to address this gap by describing how patients experience transgender clinics, as well as how these experiences compare to those experiences in other settings.

\section{METHODS}

We conducted semi-structured interviews with transgender patients. The study was approved by the Indiana University Institutional Review Board and the Eskenazi Health Research Department.

Setting. We recruited patients from the Gender Health Program of Eskenazi Health, a safety-net medical institution in central Indiana. Founded in 2016, the program is a multidisciplinary medical and surgical program that provides comprehensive, culturally competent healthcare services exclusively for transgender and gender-diverse adults. ${ }^{9}$ The sevenmembermultidisciplinary clinician team includes specialties in family medicine, plastic surgery, psychiatry, and $\mathrm{OB} / \mathrm{GYN}$ health. Additional staff in the program consists of licensed practical nurses, therapists, speech pathologists, dietitians, attorneys, medical assistant, hospital chaplain, and patient care coordinators. It is the first of its kind to serve the transgender population in Indiana and surrounding areas.

Participants. Eligible patients had at least one visit to the clinic within the last year. Participants were compensated with a $\$ 35$ gift card.

Recruitment. Before the COVID-19 pandemic, participants were recruited by letter and in person. Interested patients were directed to contact the study team. The study team then followed up with patients via phone to schedule an interview. With the onset of the COVID-19 pandemic and a dramatic decrease in on-site visits, in-person recruitment was no longer feasible. Instead, the study team recruited patients by messaging through the patient portal (MyChart). Patients who were interested in participating were directed to contact the study team.

Interviews. We first conducted individual semi-structured, hour-long interviews in person. When the COVID-19 pandemic made in-person interviews impossible, the team pivoted to conduct interviews by phone. One team member $(\mathrm{MH})$ conducted all the interviews. The interviews focused on two domains: healthcare experiences (positive and negative) and relationships with healthcare providers. As data collection progressed, we adapted our interview guide to delve more deeply into certain topics that emerged from earlier interviews, such as finding health information online.
Other Measures. Each participant also completed a short questionnaire that included questions about demographics, their use of patient portals, and their relationship with their primary care provider.

Analysis. The interviews were audio-recorded, transcribed, and analyzed using an immersion and crystallization approach. ${ }^{10}$ Recruitment continued until thematic saturation was reached. ${ }^{11,12}$ The team used NVivo software, V.12 (QSR International Pty Ltd), to organize and code interview transcripts. All transcripts and notes were independently read by two members of the research team (JLL and MH). Data were organized into meaningful units and developed into categories and themes (e.g., lack of providers) for use in representing a coherent account of participants' experiences. ${ }^{10}$ Through this iterative process, analysts organized the data in the transcripts into meaningful units, inductively developing categories for use in representing a coherent account of participants' experiences. ${ }^{13-15}$ Analysts then combined their notes and organized findings into major themes by combining similar codes and shared the findings with the greater research team. Procedures to ensure rigor and validity included practicing reflexivity (e.g., questioning interpretations, becoming aware of one's own expectations), depth of description (i.e., seeking out rich, particular details of participants' experiences), and actively seeking alternative interpretations of the data that might challenge initial study findings. ${ }^{13-15}$

\section{RESULTS}

Twenty-one patients participated in the study: eight interviews were conducted in person and 13 by phone. Table 1 summarizes patient characteristics. The mean participant age was 36 ; $76 \%$ identified as White and $43 \%$ as trans female. The demographics of our participants are similar to the county mean in race, ethnicity, and educational attainment. ${ }^{16}$ Findings are organized by three aspects of patients' healthcare experiences: limited access to care, waiting-room experiences, and encountering providers.

\section{Limited Access to Healthcare}

Study participants reported three major barriers related to access: the lack of willing providers, where they live, and long wait times.

Lack of Willing Providers. Patients described difficulty finding a provider to treat them. Some only had difficulty finding providers for transgender-specific care, while others lacked providers for any kind of healthcare. For many, this was because they lived in rural areas. For example, one patient said, 
Table 1 Participant Characteristics

\begin{tabular}{ll}
\hline \hline Characteristic & Number of patients (\%) \\
\hline Age (years) & $5(21 \%)$ \\
$18-29$ & $8(38 \%)$ \\
$30-39$ & $6(29 \%)$ \\
$40-49$ & $2(10 \%)$ \\
$50+$ & $16(76 \%)$ \\
Race & $3(14 \%)$ \\
White & $1(5 \%)$ \\
Black & $1(5 \%)$ \\
Spanish/Latino & \\
Asian & $2(10 \%)$ \\
Ethnicity & $19(90 \%)$ \\
Hispanic & $3(14 \%)$ \\
Non-Hispanic & $3(14 \%)$ \\
Gender identity & $3(14 \%)$ \\
Male & $9(43 \%)$ \\
Female & $2(10 \%)$ \\
Trans male & $1(5 \%)$ \\
Trans female & \\
Non-binary & $12(57 \%)$ \\
Other: transmasculine & $8(38 \%)$ \\
Sex assigned at birth & $1(5 \%)$ \\
Male & $1(5 \%)$ \\
Female & $13(62 \%)$ \\
Intersex & $7(33 \%)$ \\
Education attainment & $8(38 \%)$ \\
Less than high school & $9(43 \%)$ \\
High school or some college & $4(19 \%)$ \\
Bachelor's degree or higher &
\end{tabular}

I live in a very small town. [We] don't really have a lot of access to trans health plans or trans providers, because the area is so small and is so old-timey. [Patient 4]

Another said,

There was one transgender doctor in this whole county, and she retired. [Patient 16]

Although many patients commented on the difficulty of finding providers who could provide transgender-specific care, a few even had difficulty accessing care for concerns not related to transgender health. One patient was instructed to seek care elsewhere.

I went to the ER and the doctor that came in was extremely awkward... He was like, "there are people that help people like you, but I'm not one of them. I'm not sure how I can help you." I came in here for [something to do with my anxiety]. Does this require gender health knowledge? [Patient 20]

Geography. Some patients associated the limited supply of available providers with geography. The dearth of comprehensive transgender care for patients in Indiana meant that some patients had to travel long distances to receive care. One said,
I have to drive 2 to 3 hours to get [to the transgender clinic. [Patient 15]

Other patients noted that, although the transgender clinic offered primary care, obtaining such care was impractical because of how far they lived from the clinic, often an hour or more by car.

The distance from the comprehensive clinic was an especially problematic issue for patients lacking alternatives for not just transgender care, but primary care as well. One described their dilemma as follows:

[The transgender clinic physician] is more than willing to be my primary care doctor. His only concern is that he's a hundred miles away. Quite literally. It would take two hours to come see him. [...] We are going to talk about it more thoroughly at my next appointment. [...] I'm a little worried as to where I'm going to find primary care. [Patient 4]

Because many participants attributed their access issues to where they lived, some were even pleasantly surprised at being able to find, eventually, a comprehensive transgender clinic in the state. As one patient explained,

To be honest with you, when I first heard that [the transgender clinic] was happening [...], I was like is this a joke? There's no way that here in [Indiana] they're going to do this, right? [Patient 11]

In a similar vein, another patient said,

I was surprised when I moved to [Indiana] that there was a place where you could get comprehensive gender wellness care. That's been really encouraging. [Patient 19]

There were also patients who felt it was limiting that the clinic was one of their few sources for any type of healthcare:

I don't necessarily want to spend the rest of my life only going to a gender wellness clinic for my health needs. That would seriously limit my ability to go to other places in the world where those resources aren't available. It would be great if transgender practices were more integrated into general medicine practices. [Patient 19]

Wait Times. Although patients appreciated access to transgender-specific care, they sometimes faced difficulties in scheduling an appointment, facing long wait times because of high demand. Speaking of their doctor at the clinic, a patient noted, 
I know she's so busy and has so many patients that it's hard sometimes to get the appointment with her. [Patient 13]

Another patient said of the transgender clinic,

I'm glad they're expanding their staff because my wait times for some of these appointments has been for months and months. [Patient 19]

One patient said, "I had to wait six months [for my] first visit." [Patient 20]

Consequently, some who have the option choose to have a "backup" primary care provider in case they are not able to see the transgender clinic provider in a timely manner. As one patient explained,

I keep that other doctor on the back burner just in case. Let's say I get a cold or something, they can fit me in a lot faster. Rather than here, you have to schedule your appointments months out. [Patient 7]

\section{Waiting Room Experiences}

Participants described experiences with the healthcare system, staff, and other patients, by contrasting their interactions in the waiting room in non-transgender clinics with those in the comprehensive transgender clinic.

Non-transgender Clinic. For some patients, their interactions outside the transgender clinic made them uncomfortable. One shared:

It's usually the staff [who treat me weirdly]. People in the waiting area just see me as an old guy. [But the staff] see my name there and they'll look at me kind of funny and try to figure out what that means. ... [When I'm] not feeling well, that's the last thing you want to deal with. [Patient 13]

The same patient went on to describe the vulnerability of interacting with a healthcare system and not knowing when an interaction may not go well.

It didn't feel safe - not that anybody was mean or anything. It's just that you get in there and you're all nervous because now you have to explain who you are to somebody. I know they're professionals and I'm [probably] not the first trans person they ever saw. But you never know. Sometimes it's hard. [Patient 13]

Another patient described the discomfort as follows:

Being trans, it's not always comfortable sitting in a waiting room surrounded by different types of people that may or may not understand you. ... I don't feel comfortable going to anyone else that wouldn't be as openly comfortable and to treat [transgender patient]. [Patient 1]

Transgender Clinic. The discomfort felt in the nontransgender clinic waiting rooms was a contrast to the positive impression the transgender clinic left. "It's beautiful and breathtaking," one patient said of the comprehensive transgender facility, "And it feels so welcoming. Everybody is so nice and respectful, and they don't judge." [Patient 12]

Another patient noted:

Seeing people with their pronouns on their tags and just how seriously they take transgender care and transgender sensitivity is really cool and different. [Patient 18]

\section{Encountering Providers}

When it comes to individual encounters with healthcare providers, participants recounted a range of experiences, both positive and negative, with providers outside the transgender clinic. Patients only had positive experiences to share about providers from the transgender clinic.

Negative Experiences. Related to the paucity of providers willing to treat transgender patients was the small number of providers knowledgeable about transgender health. This knowledge deficit was a significant barrier to many patients, who encountered providers who looked to them for guidance on transgender care.

All I've ever done is had to educate them," one patient said with resignation, "Educate doctors. Educate pharmacists. [Patient 1]

Another patient shared,

There's also the piece of needing to explain a little bit of my identity as a trans person and having a lot of doctors like 'oh, you're my first trans patient'. I'm so glad that I can be that for you, [but] also how awkward it is for me to hear that. And a little bit nerve wracking. [Patient 2]

Some providers used their inexperience and lack of knowledge about transgender care to turn patients away:

I didn't have a primary because I'd never felt comfortable... When I would go to urgent care it would have to be really for something because I hardly get sick. Even then, it's like, "Oh, we're not sure because you're trans. This is different to us. We don't know how to treat this. [Patient 1]

Positive Experiences with Non-transgender Clinic Providers. There were a few exceptions to the participants' 
negative experiences with providers not part of the transgender clinic. Some participants had strong relationships with the providers despite the providers' lack of knowledge about transgender health. One, for example, spoke of a long-time relationship with a primary care provider who provided primary care that was not specific to transgender health:

[I've seen this doctor for] 17 years. She's too good of a doctor to let go. She said she has no problems handling everything else. She just doesn't know about [transgender health], so she wasn't going to do something she didn't know. [Patient 21]

Positive Experiences with Transgender Clinic Providers. In contrast to providers outside of the transgender clinic, patients described their encounters with physicians in the transgender clinic in overwhelmingly positive and effusive terms. Several specifically mentioned trust as a key element of their experiences with the providers in the transgender clinic.

I could wax poetic about Dr. [Name] for days... She is the first doctor that I fully trust. She says something to me, I take it to the bank. ...I'm extremely critical of the medical profession ... but I trust her implicitly. You can see it in her face, you can hear it in the way that she speaks that she legitimately gives a crap. [Patient 20]

I feel like it's mutual... I trust her authority to tell me it needs to be this or let's wait this amount of time and then revisit it. I feel I can trust that once I address something, she'll have a plan for me. [Patient 18]

Another theme of the positive experiences centered around acceptance by their providers, including non-verbal cues like being treated in a manner consistent with the gender with which they identify:

He doesn't make me feel like I'm a man coming into the transgender clinic. He makes me feel like I'm wanted. He talks to me as if I'm a woman. ... It just feels like a regular doctor's appointment [where] I'm going to go get checked up. [Patient 17]

This sentiment was echoed by another patient, who said,

She always sees me as a woman. It's always the right pronouns. She's got everyone in that office now with the right pronouns. ... I really feel like she champions the trans community and that means a lot to me. [Patient 13]

One patient spoke of being able to be open in communicating with their provider because they did not feel judged:
I can talk to him about anything and he's not going to judge me. Not going to make me feel like [...] did I say too much or not enough? [Patient 17]

Finally, patients also described appreciating how the providers at the clinic communicated with them. One patient said,

They're really honest and, and open and straightforward. They don't beat around the bush. ...They're always polite and caring." [Patient 20]

[My interaction with my doctor] made me feel like I was actually going to be listened to when I spoke to him and that he was actually hearing and listening to what I was saying." [Patient 10]

Even when disagreements occurred, patients described feeling genuinely cared for, as with the following patient:

I think Dr. [Name] is a little more conservative than I like of my [hormone] treatments. ... She gets her way every time. I always give in, but it's because she cares so much. I get it. She wants to make sure I'm OK. [Patient 13]

\section{DISCUSSION}

Our interviews with patients from a transgender clinic in the Midwest revealed a deficit in the number of providers and quality of care to which transgender patients have access. Some patients had difficulty finding providers who were knowledgeable about hormone treatments and transgender care; others had trouble finding providers for any type of care. Outside the comprehensive transgender care setting, patients had difficulty accessing care and finding willing and knowledgeable providers. Within the transgender clinic, patients found staff and providers who were knowledgeable and whom they felt that they could trust. While there were some advantages to obtaining healthcare from a clinic focused specifically on transgender individuals and transgender health, some patients nonetheless indicated that the clinic could not address all of their healthcare needs. While there are few published data on such clinics in the USA, they have gained more prominence over the past decade. Our study fills a gap in the literature by describing the experiences and healthcare needs of transgender patients in the Midwest who seek care at a comprehensive transgender clinic. More research is needed to understand their effectiveness in this population.

The dearth of primary care providers capable of delivering care described by our study participants is aligned with what is known about physician education on transgender care. Among medical schools in the USA and Canada, a median of $5 \mathrm{~h}$ is dedicated to teaching lesbian, gay, bisexual, transgender, and 
queer-related(LGBTQ-related) content. ${ }^{17} \mathrm{Few}$ teach transgender-specific content, and a third of programs reportedly spend no time on LGBTQ health-related content during clinical training. ${ }^{17}$ Despite the absence of formal teaching in many medical schools, in two recent surveys, physicians reported relatively high confidence in their ability to care for transgender patients, and not feeling as if they need education. In one study, only $41 \%$ of survey respondents within a rural tertiary care centered agreed that they needed more education about transgender patients to provide appropriate care. The great majority agreed that they were willing to, and comfortable with, treating transgender patients within their scope of practice. ${ }^{18}$ In another survey, more than half of respondents reported having formal education about transgender and gender-diverse care, and being capable of providing routine care to transgender patients. ${ }^{19,20}$ In contrast, the data from this study revealed some gaps in the care of transgender patients. Future research on provider experiences with delivering transgender care may help to explain the potential mismatch of perceptions between patients and physicians.

Beyond individual encounters with providers, participants in our study also recounted positive and negative perceptions of the healthcare environment at the comprehensive transgender clinic and other outside clinics. They noted their feelings related to those spaces, describing non-transgender-specific waiting areas and experiences with the front desk staff and fellow patients as "not always comfortable." In contrast, the transgender clinic felt "so welcoming." In describing the healthcare experiences of another marginalized group-Black veterans in the VA-Eliacin et al. described patients' perceptions of non-verbal cues, such as workforce diversity and socially inclusive art displays, in indicating to minority patients whether they will be welcomed. ${ }^{21}$ In the present study, many factors may have accounted for patients' discomfort in some healthcare settings, and comfort in others: the welcome that the comprehensive transgender clinic engenders may be due to the physical environment (e.g., displays inclusive of transgender patients, identification badges with pronouns), the behaviors of the clinic staff (e.g., use of desired pronouns), or merely the patients' identification of the clinic as a safe space, from word of mouth or personal experience. Contextual factors, such as an individual's knowledge of, and personal experiences with, bias, can shape perceptions of bias ${ }^{21-23}$, and the need for providers and healthcare systems to seek to reduce systemic bias and create a welcoming environment. ${ }^{24,}{ }^{25}$ In building a welcoming healthcare environment for transgender patients, policymakers and healthcare providers must be attuned to non-verbal cues, as well as the experiences that their patients bring.

Participants often spoke of positive care that they received from the transgender clinic in emotive ways. They felt seen, heard, and understood by their providers. In contrast, participants had a mix of experiences with providers outside the transgender clinic. Although some experiences were positive, many patients faced providers either unable or unwilling to provide care. These experiences are consistent with previous studies about transgender patients' experiences in nontransgender clinic settings, including inconsistent access and even refusal of services. ${ }^{8,26,27}$ Few interventions have been tested that attempt to mitigate these negative experiences: the focus of interventions in this area has often been on provider education and the creation of comprehensive transgender health clinics. ${ }^{5,28-30}$ Although education can improve providers' knowledge, its long-term effectiveness in improving patient experiences is not certain. In the meantime, too many transgender patients such as those from this study, especially those living in rural areas, lack reliable access to competent primary care that is free of bias.

This study contributes to the emerging literature about the experiences of patients with comprehensive transgender clinics as well as the experiences of those patients in other settings. Provider practices such as using correct pronouns, being knowledgeable, and not judging patient lifestyles made patients feel that they could trust their doctors. These findings reflect transgender patient surveys of patients highly satisfied with their care at the same clinic. ${ }^{9}$ At the same time, the patient experiences also reflect the deficits of many clinics and providers in caring for transgender patients. These deficits suggest the need for interventions beyond clinical education. Training providers and their staff in practices that would make transgender patients feel safe and welcome, such as using proper pronouns, could help address these deficits, and deficits in primary care options for transgender patients. Our study also noted the limitation of the comprehensive clinic, particularly related to geography and high demand for comprehensive transgender health services. While the clinic is adding providers to expand its capacity, it cannot meet the needs of the whole state. The high patient demand demonstrates both the need for, and success of, comprehensive clinics, as well as the need for alternative solutions, particularly those that build provider capacity in areas without such clinics. These could include models of care that have worked for other vulnerable populations in similar settings, such as tele-consultations for providers, or increasing the availability of video visits for patients. $^{17,18,20,31}$

Limitations. Our study focused on the experiences of patients who had received care at a comprehensive transgender clinic in the Midwest. Because healthcare experiences depend on context, our findings might not be generalizable to other settings. The US transgender population is ethnically and geographically diverse. ${ }^{32,} 33$ Transgender patients in other locales, with different available resources, may have different experiences with different needs. Our study might also not represent transgender patients who choose not to seek care at a transgender-specific clinic. Even with these limitations, our study was able to capture the experiences of participants experiencing a range of healthcare settings, with providers with a range of experiences with transgender 
health. In gathering data until we reached theme saturation, we present findings that may reflect those of a broader group whose experiences with transgender clinics have not previously been described.

\section{CONCLUSION}

This study adds to the still nascent literature about comprehensive transgender clinics, by focusing on the lived healthcare experiences of the patients that seek care there; it speaks to the need for primary care providers who can treat transgender patients. The study also reinforces the need for healthcare spaces that feel safe to transgender patents while advancing our understanding of the experiences of transgender patients in the Midwest. Outside specialty transgender settings, many patients had negative experiences with providers who were unwilling or unable to provide care. In the dedicated transgender care setting, patients had positive encounters with providers, and built trusting relationships, yet some also faced long wait times and had to travel great distances to access care. Overall, transgender patients expressed a need for healthcare services, particularly for primary care, that are partially met by the comprehensive care clinic model. These findings set the stage for future interventions aimed at expanding the capacity of providers and health systems to address the healthcare needs of transgender patients.

Supplementary Information The online version contains supplementary material available at https://doi.org/10.1007/s11606-02207408-5.

Acknowledgements: Dr. Weiner is Chief of Health Services Research and Development at the Richard L. Roudebush Veterans Affairs Medical Center in Indianapolis, Indiana. The views expressed in this article are those of the authors and do not necessarily represent the views of the U.S. Department of Veterans Affairs.

Corresponding Author: Joy L. Lee, PhD, MS; Regenstrief Institute, Inc., Indianapolis, IN, USA (e-mail: joyllee@iu.edu).

Author Contribution Study concept and design: JLL, MSM, MW, JMF

Acquisition of subjects and/or data: $M H$, JLL

Analysis and interpretation of data: JLL, NAR, JLC, JDF, JMF, MSM

Preparation of manuscript: JLL, MW, MSM

\section{Declarations:}

Conflict of Interest: The authors declare that they do not have a conflict of interest.

\section{REFERENCES}

1. Downing, J. M., \& Przedworski, J. M. (2018). Health of transgender adults in the U.S., 2014-2016 Am J Preven Med, 55(3), 336-344. https://doi.org/10.1016/j.amepre.2018.04.045

2. Dragon, C. N., Guerino, P., Ewald, E., \& Laffan, A. M. (2017). Transgender Medicare beneficiaries and chronic conditions: Exploring fee-for-service claims data. LGBT Health, 4(6), 404-411. https://doi.org/ 10.1089/lgbt.2016.0208.
3. Herman $\mathbf{J}$, Wilson BD, Becker T. Demographic and Health Characteristics of Transgender Adults in California: Findings from the 2015-2016 California health interview survey. Policy Brief UCLA Cent Health Policy Res 2017(8):1-10. (https://www.ncbi.nlm.nih.gov/pubmed/29091375).

4. James, S. E., Herman, J. L., Rankin, S., Keisling, M., Mottet, L., \& Anafi, M. (2016). The report of the 2015 U.S. transgender survey. National Center for Transgender Quality: Washington, D.C.

5. Kaigle, A., Sawan-Garcia, R., \& Firek, A. (2017). Approach to the provision of transgender health care in a veteran population. Ment Health Clin, 7(4), 176-180. https://doi.org/10.9740/mhc.2017.07.176.

6. Shipherd, J. C., Kauth, M. R., \& Matza, A. (2016). Nationwide interdisciplinary E-consultation on transgender Care in the Veterans Health Administration. Telemed J e-health : The Off J Am telemed Assoc, 22(12), 1008-1012. https://doi.org/10.1089/tmj.2016.0013.

7. Bockting, W., Robinson, B., Benner, A., \& Scheltema, K. (2004). Patient satisfaction with transgender health services. J Sex Marital Therapy, 30(4), 277-294. https://doi.org/10.1080/ 00926230490422467

8. De Santis, J. P., Cintulova, M., Provencio-Vasquez, E., Rodriguez, A. E., \& Cicero, E. C. (2020). Transgender women's satisfaction with healthcare services: A mixed-methods pilot study. Perspectives Psychiatric Care, 56(4), 926-938. https://doi.org/10.1111/ppc.12514.

9. Nowaskie, D. Z., Fogel, R. S., \& Fogel, J. M. (2019). Impact on patient satisfaction and importance of medical intake and office staff in a multidisciplinary, one-stop shop transgender program in Indianapolis, Indiana. J Multidiscipl Healthcare, 12, 665-673. https://doi.org/10. 2147/JMDH.S216969.

10. Borkan J. Immersion/Crystallization. In: Crabtree BF, Miller WL, eds. Doing Qualitative Research. Thousand Oaks, California: Sage Publications, Inc.; 1999:179-194.

11. Charmaz $\mathbf{K}$. Theoretical sampling, saturation, and sorting. Constructing grounded theory: Sage; 2014:192-224.

12. Vasileiou, K., Barnett, J., Thorpe, S., \& Young, T. (2018). Characterising and justifying sample size sufficiency in interview-based studies: Systematic analysis of qualitative health research over a 15-year period. BMC Med Res Methodol, 18(1), 148. https://doi.org/10.1186/ s12874-018-0594-7.

13. Bernard, H. (2002). Research methods in anthropology: Qualitative and quantitative approaches. Walnut Creek, CA: AltaMira Press.

14. Miles, M., \& Huberman, A. (1994). Qualitative data analysis. Thousand Oaks, CA: Sage.

15. Miller, W. L., \& Crabtree, B. F. (1999). The dance of interpretation. In B. F. Crabtree \& W. L. Miller (Eds.), Doing qualitative research (pp. 179-194). Thousand Oaks, CA: Sage.

16. United States Census Bureau. QuickFacts: Indianapolis city (balance), Indiana. (https://www.census.gov/quickfacts/fact/table/ IN, indianapoliscitybalanceindiana\#).

17. Obedin-Maliver, J., Goldsmith, E. S., Stewart, L., et al. (2011). Lesbian, gay, bisexual, and transgender-related content in undergraduate medical education. Jama, 306(9), 971-977. https://doi.org/10. 1001/jama.2011.1255

18. Rowan, S. P., Lilly, C. L., Shapiro, R. E., et al. (2019). Knowledge and attitudes of health care providers toward transgender patients within a rural tertiary care center. Transgend Health, 4(1), 24-34. https://doi.org/ $10.1089 / \operatorname{trgh} .2018 .0050$.

19. Stroumsa, D., Shires, D. A., Richardson, C. R., Jaffee, K. D., \& Woodford, M. R. (2019). Transphobia rather than education predicts provider knowledge of transgender health care. Med Educ, 53(4), 398407. https://doi.org/10.1111/medu.13796.

20. Shires, D. A., Stroumsa, D., Jaffee, K. D., \& Woodford, M. R. (2018). Primary care Clinicians' willingness to Care for Transgender Patients. Ann Fam Med, 16(6), 555-558. https://doi.org/10.1370/afm.2298.

21. Eliacin, J., Matthias, M. S., Cunningham, B., \& Burgess, D. J. (2020). Veterans' perceptions of racial bias in VA mental healthcare and their impacts on patient engagement and patient-provider communication. Patient Educ Counseling, 103(9), 1798-1804. https://doi.org/10.1016/j. pec.2020.03.017.

22. Henderson, J., Gao, H., \& Redshaw, M. (2013). Experiencing maternity care: The care received and perceptions of women from different ethnic groups. BMC Pregnancy Childbirth, 13, 196. https://doi.org/10.1186/ 1471-2393-13-196.

23. Plaut, V. C., Garnett, F. G., Buffardi, L. E., \& Sanchez-Burks, J. (2011). "what about me?" perceptions of exclusion and whites' reactions to multiculturalism. J Personal soc psychol, 101(2), 337-353. https://doi. org/10.1037/a0022832 
24. Phelan, S. M., Burke, S. E., Hardeman, R. R., et al. (2017). Medical school factors associated with changes in implicit and explicit bias against gay and lesbian people among 3492 graduating medical students. J Gen Int Med, 32(11), 1193-1201. https://doi.org/10.1007/s11606017-4127-6.

25. Tan, J. Y., Baig, A. A., \& Chin, M. H. (2017). High stakes for the health of sexual and gender minority patients of color. J Gen Int Med, 32(12), 1390-1395. https://doi.org/10.1007/s11606-017-4138-3.

26. Howard, S. D., Lee, K. L., Nathan, A. G., Wenger, H. C., Chin, M. H., \& Cook, S. C. (2019). Healthcare experiences of transgender people of color J Gen Int Med, 34(10), 2068-2074. https://doi.org/10.1007/s11606019-05179-0.

27. Johnson, A. H., Hill, I., Beach-Ferrara, J., Rogers, B. A., \& Bradford, A. (2020). Common barriers to healthcare for transgender people in the U.S. southeast. Int J Transgend Health, 21(1), 70-78. https://doi.org/10. 1080/15532739.2019.1700203.

28. Dubin, S. N., Nolan, I. T., Streed, C. G. Jr., Greene, R. E., Radix, A. E. \& Morrison, S. D. (2018). Transgender health care: Improving medical students' and residents' training and awareness. Adv Med Educ Pract, 9 377-391. https://doi.org/10.2147/AMEP.S147183.

29. McCave EL, Aptaker D, Hartmann KD, Zucconi R. Promoting Affirmative Transgender Health Care Practice Within Hospitals: An IPE Standardized Patient Simulation for Graduate Health Care Learners. MedEdPORTAL 2019;15:10861. https://doi.org/10.15766/mep_23748265.10861.
30. Thomas, D. D., \& Safer, J. D. (2015). A simple intervention raised resident-physician willingness to assist transgender patients seeking hormone therapy. Endocrine Pract, 21(10), 1134-1142. https://doi.org/ 10.4158/EP15777.OR

31. Magnus, M., Edwards, E., Dright, A., et al. (2020). Development of a telehealth intervention to promote care-seeking among transgender women of color in Washington. DC. Public Health Nurs, 37(2), 262-271. https://doi.org/10.1111/phn. 12709.

32. Cicero, E. C., Reisner, S. L., Silva, S. G., Merwin, E. I., \& Humphreys J. C. (2019). Health care experiences of transgender adults: An integrated mixed research literature review. ANS. Advanc Nursing Sci, 42(2), 123138. https://doi.org/10.1097/ANS.0000000000000256.

33. Feldman, J., Brown, G. R., Deutsch, M. B., et al. (2016). Priorities for transgender medical and healthcare research. Curr Opin Endocrinol, Diabetes, Obesity, 23(2), 180-187. https://doi.org/10.1097/MED. 0000000000000231.

Publisher's Note: Springer Nature remains neutral with regard to jurisdictional claims in published maps and institutional affiliations. 